\title{
High chromosomal instability in adenocarcinoma of the ileum arising from multifocal gastric heterotopia with gastritis cystica profunda
}

\author{
Inga-Marie Schaefer · Philipp Schüler • Christina Enders • \\ Jens-Gerd Scharf • Silke Cameron · Giuliano Ramadori • \\ László Füzesi
}

Received: 28 May 2010/Accepted: 14 June 2010/Published online: 25 June 2010

(C) Springer Science+Business Media, LLC 2010

\begin{abstract}
Adenocarcinoma of the small intestine arising from heterotopic gastric mucosa is extremely rare. In this report, we present the case of a 68-year-old woman who complained of abdominal pain, weight loss and subileus. Gross examination of resected small bowel revealed multiple flat polypous lesions with cysts in the ileal submucosa, one of which containing an ulcerated, stenosing tumour. On microscopic examination, an adenocarcinoma of the ileum arising from multifocal gastric heterotopia with secondary gastritis cystica profunda was diagnosed. Comparative genomic hybridization of the adenocarcinoma revealed chromosomal gains at 1q, 3q, 5p, 8q, 11p, 12p, $13 q$ and losses at Xp, 4q, 8p, 10p, 14q, 17p, 20p, compatible with a high degree of genomic instability.
\end{abstract}

Informed consent Written informed consent was obtained from the patient. A copy of the written consent is available for review by the Editor-in-Chief of this journal.

I.-M. Schaefer $(\varangle) \cdot$ C. Enders · L. Füzesi

Department of Gastroenteropathology, University Medical

Center Göttingen, Robert-Koch-Straße 40, 37075 Göttingen,

Germany

e-mail: schaeferinga@web.de

P. Schüler

Department of General and Visceral Surgery, University

Medical Center Göttingen, Göttingen, Germany

J.-G. Scharf · S. Cameron · G. Ramadori

Department of Gastroenterology and Endocrinology, University

Medical Center Göttingen, Göttingen, Germany
Keywords Gastric heterotopia .

Gastritis cystica profunda · Adenocarcinoma · Ileum ·

Comparative genomic hybridization

\section{Introduction}

Despite the fact that the small intestine accounts for $90 \%$ of mucosal surface of the gastrointestinal tract, adenocarcinomas of the small bowel make up less than $5 \%$ of all gastrointestinal malignancies [1]. Risk factors include male gender, age, non-Asian ethnicity, familial adenomatous polyposis (FAP), hereditary non-polyposis colorectal cancer (HNPCC) and Crohn's disease [1]. We report a case of multifocal gastric heterotopia with gastritis cystica profunda in the ileum and an adenocarcinoma in one of these lesions. Adenocarcinoma arising from heterotopic gastric mucosa is very infrequent. To date, only one similar case in the jejunum has been reported [2].

\section{Case report}

A 68-year-old woman was admitted to hospital with a sixweek history of abdominal pain and 6-kg weight loss. Her medical history revealed arterial hypertension, diabetes mellitus type II, sigma diverticulosis, cholecystectomy and hysterectomy. Family history was uneventful for bowel diseases. Esophagogastroscopy and colonoscopy detected no pathologies. Abdominal computed tomography demonstrated a circular stenosis of the distal ileum (Fig. 1a). Tumourous obstructive ileus was suspected, and explorative laparotomy was performed. Subtotal stenosis of the distal ileum and numerous small polypous lesions were detected in the small bowel. Thirty nine centimetres 

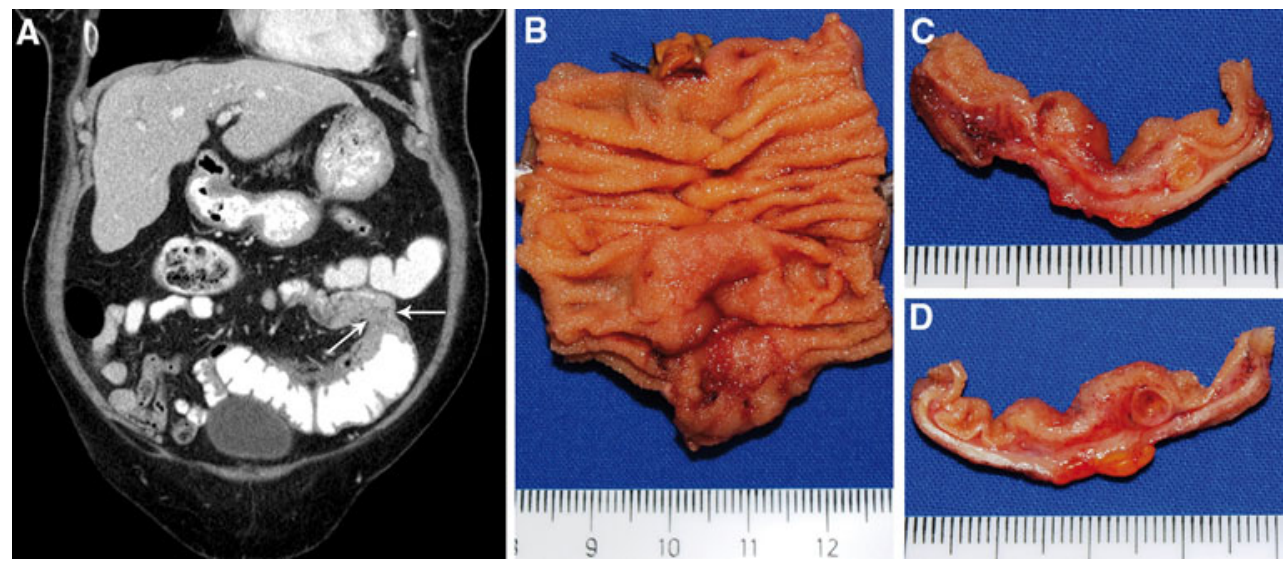

Fig. 1 Preoperative abdominal computed tomography and gross findings. A stenosing tumour of the small intestine (arrows) was detected in computed tomography scan (a). Gross pathological findings of the resected specimen demonstrated an ulcerated tumour of the ileum (b) and cross-sections of numerous polypous lesions, displaying cystic dilations located mostly in mucosa and submucosa $(\mathbf{c}, \mathbf{d})$
Fig. 2 Histopathological findings of the resected small bowel specimen. Haematoxylineosin (HE) staining showed normal ileal mucosa adjacent to heterotopic gastric corpus mucosa with cystic dilation of the submucosal gastric glands similar to gastritis cystica profunda $(\mathbf{a}, \times 20)$. Focal development of intraepithelial neoplasia $(\mathbf{b}, \times 100)$ and invasive adenocarcinoma was detected in an area of gastric heterotopia $(\mathbf{c}, \times 100)$
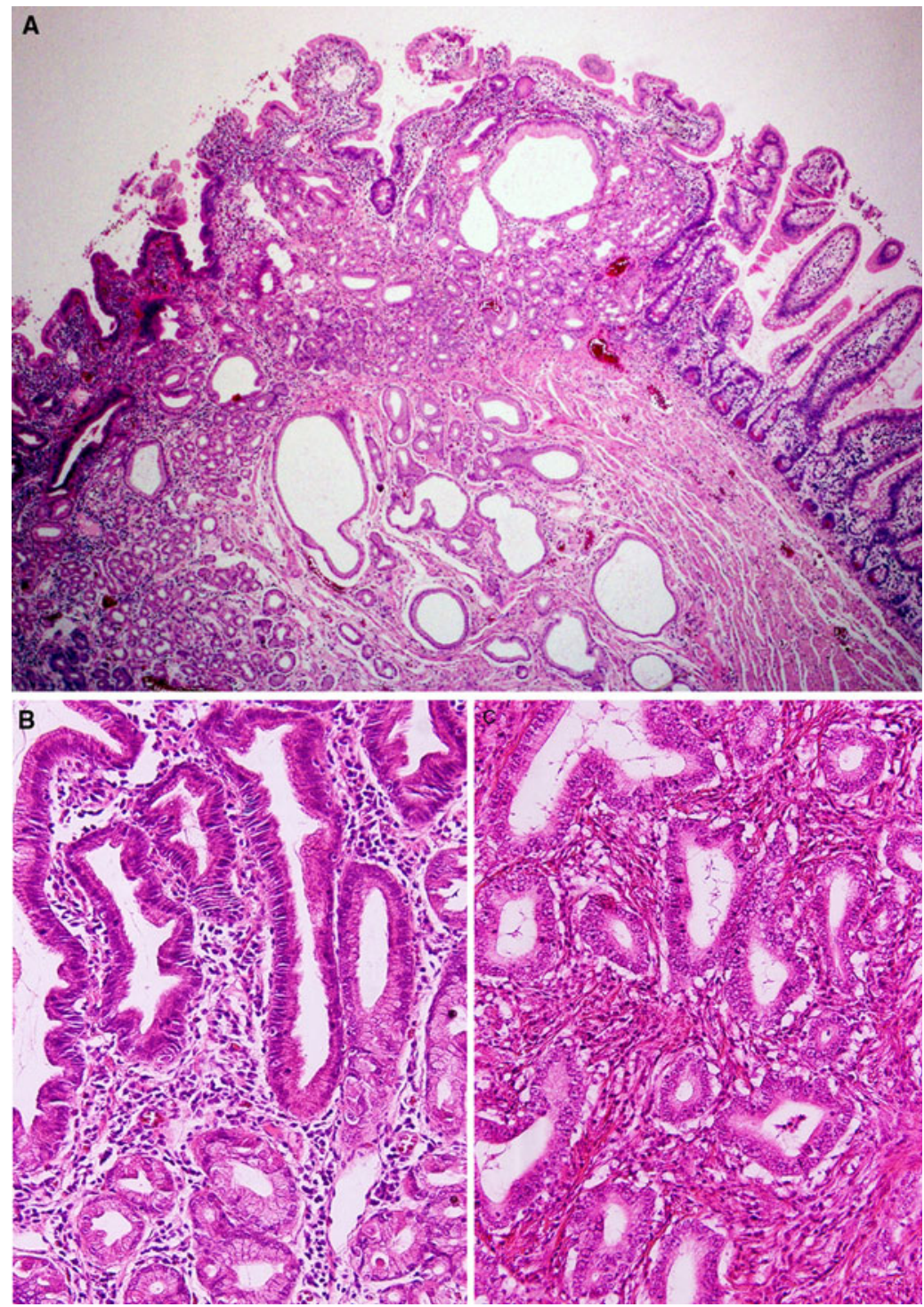
of small bowel was resected and displayed a central 4-cm circular stenosing, ulcerated tumour exhibiting a cystic cut surface. Furthermore, numerous smaller flat polypous lesions up to $3 \times 2 \mathrm{~cm}$ were detected throughout the specimen displaying a central folded surface and cystic cross-section (Fig. 1b-d). On microscopic examination, ileal mucosa was interrupted by patchy glandular structures of gastric corpus and antrum differentiation, located mostly in the mucosa (Fig. 2a). Intraepithelial neoplasia was present in mucosa overlying heterotopic corpus glands (Fig. 2b). Cystic dilation of submucosal glands was observed corresponding to gastritis cystica profunda. In addition, an adenocarcinoma was originated in the heterotopia with submucosal infiltration (Fig. 2c). Immunohistochemical staining with cytokeratin (CK) 7 was positive in heterotopic glands, and CK20 stained positive in ileal mucosa. The adenocarcinoma displayed only CK7. Proliferative activity assessed by Ki67 was estimated at $60 \%$ in the adenocarcinoma. Finally, the diagnosis of multifocal heterotopic gastric mucosa in the distal ileum with gastritis cystica profunda and development of a $4-\mathrm{cm}$ invasive adenocarcinoma was established and staged at pT3, pN0 (0/12), pMx, G2, R0 according to UICC 2010.
Postoperative capsule endoscopy did not detect any more pathologies.

CGH from formalin-fixed and paraffin-embedded specimens of the adenocarcinoma was performed as described previously and revealed ish $\operatorname{cgh} \operatorname{enh}(1)(q), \operatorname{enh}(3)(q), \operatorname{dim}(4)(q)$, $\operatorname{enh}(5)(p), \operatorname{dim}(8)(p), \operatorname{enh}(8)(q), \operatorname{dim}(10)(p), \operatorname{enh}(11)(p), \operatorname{enh}(12)$ $(p), \operatorname{enh}(13)(q), \operatorname{dim}(14)(q), \operatorname{dim}(17)(p), \operatorname{dim}(20)(p), \operatorname{dim}(X)(p)$ (Fig. 3) [3].

\section{Discussion}

Heterotopic gastric mucosa is fairly infrequent but may be encountered throughout the gastrointestinal tract, usually as "inlet patch" in proximal oesophagus, duodenum or Meckel's diverticulum [4]. The peculiar observation in our case was not only the multifocal gastric heterotopia but the combination with gastritis cystica profunda. The latter is characterized by hyperplastic and cystic dilation of pseudopyloric glands with extension into the submucosa or even muscular wall of the stomach. Its aetiology is still unclear. Ischaemia, chronic inflammation, mucosal injury, the effects of surgery and suture material are suggested to

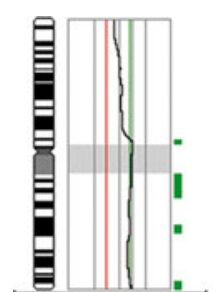

$1(27)$

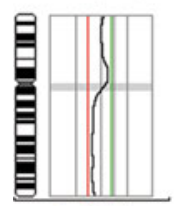

$6(31)$
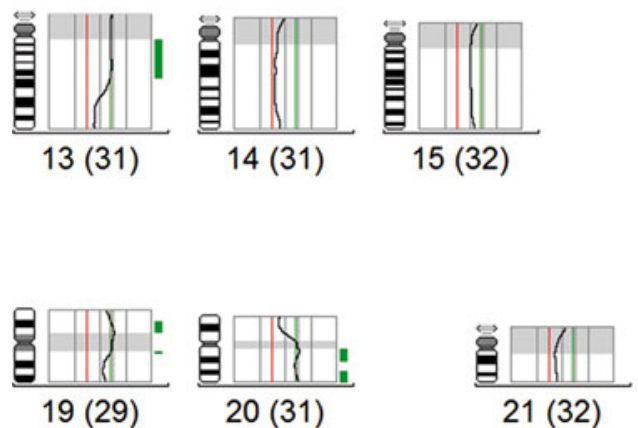

Fig. 3 Comparative genomic hybridization $(\mathrm{CGH})$ of the ileal adenocarcinoma revealed ish $\operatorname{cgh} \operatorname{enh}(1)(q), \operatorname{enh}(3)(q), \operatorname{dim}(4)(q), \operatorname{enh}(5)$ $(p), \operatorname{dim}(8)(p), e n h(8)(q), \operatorname{dim}(10)(p), e n h(11)(p), e n h(12)(p), e n h(13)(q)$, $\operatorname{dim}(14)(\mathrm{q}), \operatorname{dim}(17)(\mathrm{p}), \operatorname{dim}(20)(\mathrm{p}), \operatorname{dim}(\mathrm{X})(\mathrm{p})$ as indicated by red bars
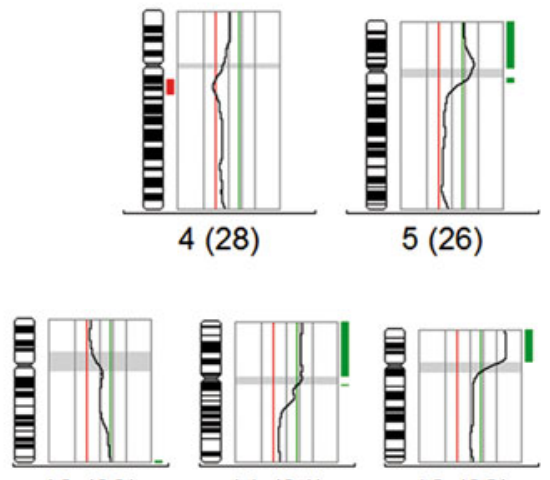

$10(33)$

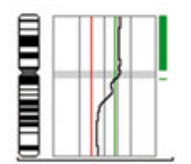

$11(34)$
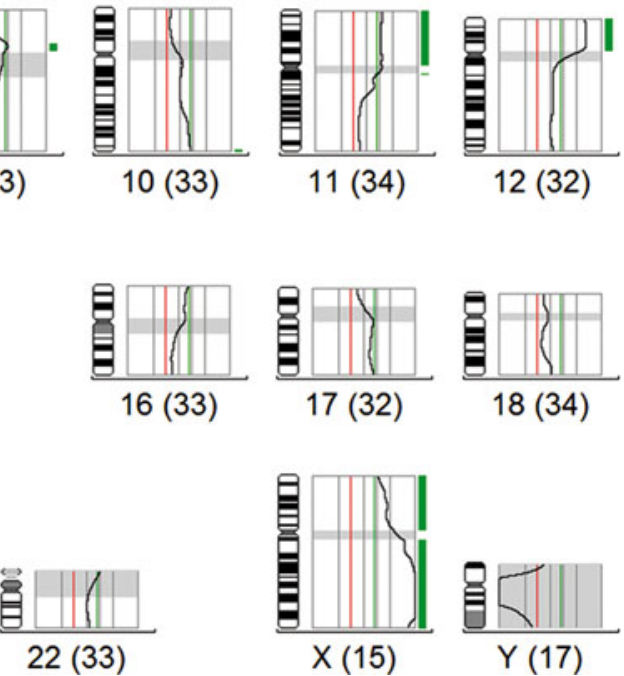

on the left and green bars on the left of the ideograms. The number of chromosomes included in the CGH analysis is indicated at the bottom of each individual profile 
play a role in pathogenesis [5]. Gastritis cystica profunda is a benign lesion, although a possible precancerous nature has been hypothesized [5].

Malignant transformation of heterotopic gastric mucosa is described for oesophagus, gallbladder and adenomyosis of the stomach. In our case, adenocarcinoma developed within the heterotopia, giving rise to the question of gastric or intestinal origin. The immunostaining of $\mathrm{CK} 7+/ 20-$ multifocal heterotopic gastric mucosa complementary to CK7-/20+ ileal mucosa favours a gastric origin of the tumour, although intestinal mucosa may sometimes be CK7+/CK20- as well.

Our CGH results revealed a high degree of genomic instability in the adenocarcinoma, but except for gains on $8 \mathrm{q}$ and losses on $4 \mathrm{q}$, they differed from previous findings on intestinal type gastric cancer [6].

The present case highlights the fact that heterotopic gastric mucosa of the small intestine possesses malignant potential and requires close clinical monitoring when diagnosed.

Acknowledgments We thank Prof. Jörg Männer from the Department of Anatomy and Embryology, University Medical Center, Göttingen, Germany, for his scientific support.
Conflict of interests The authors declare that they have no conflict of interests.

\section{References}

1. Planck M, Ericson K, Piotrowska Z, Halvarsson B, Rambech E, Nilbert M. Microsatellite instability and expression of MLH1 and MSH2 in carcinomas of the small intestine. Cancer. 2003;97: 1551-7.

2. Caruso ML, Marzullo F. Jejunal adenocarcinoma in congenital heterotopic gastric mucosa. J Clin Gastroenterol. 1988;10:92-4.

3. Gunawan B, Schulten HJ, von HA, Schmidt B, Enders C, Hoer J, Langer C, Schuler P, Schindler CG, Kuhlgatz J. Site-independent prognostic value of chromosome $9 \mathrm{q}$ loss in primary gastrointestinal stromal tumours. J Pathol. 2004;202:421-9.

4. Alagozlu H, Ergun M, Cindoruk M, Unal S, Dumlu S, Poyraz A, Dursun A. The rare presentations of a large polyp and an esophageal carcinoma in heterotopic gastric mucosa: a case series. J Med Case Reports. 2007;1:127.

5. Franzin G, Musola R, Zamboni G, Manfrini C. Gastritis cystica polyposa: a possible precancerous lesion. Tumori. 1985;71:13-8.

6. Kokkola A, Monni O, Puolakkainen P, Larramendy ML, Victorzon M, Nordling S, Haapiainen R, Kivilaakso E, Knuutila S. 17q12-21 amplicon, a novel recurrent genetic change in intestinal type of gastric carcinoma: a comparative genomic hybridization study. Genes Chromosomes Cancer. 1997;20:38-43. 